\title{
A study of many-year spectral variability of the Ae Herbig star HD 36112
}

\author{
M.I. Fursyak, O.V. Kozlova \\ Crimean Astrophysical Observatory, Nauchny, Crimea, 298409 \\ e-mail: mbjhht@yandex.ru
}

Submitted on October 7, 2020

\begin{abstract}
We present the results of long-term high-dispersion spectral observations ( $\mathrm{R}=20000)$ of the Ae Herbig star HD 36112 in the regions of the $\mathrm{H}_{\alpha}$ emission line and the NaI D resonance doublet lines. They show that parameters of the $\mathrm{H}_{\alpha}$ emission line demonstrate complicated variability on several time scales: 1) variability from night to night caused by inhomogeneity of the circumstellar envelope; 2) variability on a time scale of about $1200^{d}$ characterized by a variation of the equivalent width, intensity, and other emission parameters; 3) variability on a time scale of more than $4000^{d}$ observed as a many-year trend in variations of parameters of the $\mathrm{H}_{\alpha}$ emission line.

We associate these results with variability of physical and kinematic conditions in the inner regions of the accretion disk and wind. The most probable mechanism of this variability is a process of planet formation in the circumstellar disk.
\end{abstract}

Key words: Ae Herbig stars, spectral variability, planetary formation, wind, circumstellar disks

\section{Introduction}

The star HD 36112 (MWC 758) is attributed to a group of young hot intermediate-mass stars or the Ae/Be Herbig stars. They are surrounded by the remnants of host clouds representing the extended gas and dust disks with ongoing active processes of planet formation. Accretion of the circumstellar (CS) matter on the star is known to occur in its inner regions, whereas the processes of gas-wind outflows are observed at high latitudes (see, e.g., Alecian et al., 2013). Therefore, the Ae/Be Herbig stars are characterized by the presence in their spectra of CS emission lines, which are formed in the gaseous envelope, as well as by the IR excesses associated with circumstellar dust radiation. This is true with regard to HD 36112.

Although the star is in the known Taurus-Auriga starforming region, it was attributed to young stars only in the 1990s with the detection of IR excesses in it (The et al., 1994). According to Palla and Stahler (1993), the stellar mass is 1.8 solar masses, while its age is about 5-10 million years, which is close to the age estimates of other Ae/Be Herbig stars. The spectral type of the star is A8Ve, brightness in the $\mathrm{V}$ band is $8^{m} .3$, whereas the axial rotation period is about $1.8^{d}$ (Beskrovnaya et al., 1999).

The photometric studies of HD 36112 are quite fragmented. This is caused by its weak photometric activity (the variability amplitude does not exceed several tenths of stellar magnitude following Beskrovnaya et al. (1999)) and associated with the orientation of the star with respect to an observer. Thus, from the IR interferometry performed by Eisner et al. (2004) and Isella et al. (2008), the inclination angle of the rotation axis of HD 36112 to the line-of-sight accounts for about $30-40^{\circ}$. This means that the star is oriented at the intermediate angle, at which the wind region emerges on the line-of-sight (Grinin, Rostopchina, 1996).

A detailed analysis of fast spectral variability of HD 36112 was carried out in Pogodin (1995) and Beskrovnaya et al. (1999). The results of these studies showed that the CS emission lines in a spectrum have a $\mathrm{P}$ Cyg-type profile, which is characteristic of outflowing gas. The authors associated the strong variability of parameters of emission lines from night to night with the presence of azimuthal inhomogeneities in the wind structure. Unfortunately, no data are available on stellar spectral variability on other time scales.

Recent studies of the HD 36112 disk structure in the millimeter and near IR ranges (Boehler et al., 2018; Benisty et al., 2015) showed that a young planet system is actively formed in the circumstellar disk of HD 36112. Since the modeling data show that the planet formation process (coupled with binarity) is able to not just change the structure of disks but produce a variation of the accretion rate on a time scale that is equal to the orbital period (see Demidova, 2016, 2009), we attempted to ascertain the observed signatures of such variations using the data of many-year spectral monitoring of HD 36112 conducted at the Crimean Astrophysical Observatory.

\section{Observations}

The high-resolution spectra of HD $36112(\mathrm{R}=20000)$ were derived with the 2.6-meter telescope (ZTSH) at CrAO between 2009 and 2018. By 2013, the spectra were taken with 
the coude spectrograph ASP-14 and CCD camera. Since 2013, the observations have been carried out with the echelle spectrograph ESPL mounted at the coude focus. Fifty-three spectra were totally acquired in the region of the $\mathrm{H}_{\alpha}$ emission line and 13 spectra in the region of the $\mathrm{NaI} \mathrm{D}$ resonance doublet lines.

The processing of spectra was performed using the SPE software package developed by S.G. Sergeev and used at CrAO.

When determining parameters of the $\mathrm{H}_{\alpha}$ emission line and the $\mathrm{NaI} \mathrm{D}$ resonance doublet, we subtracted the synthetic spectrum from the observed one (such a practice is widely accepted when analyzing emission profiles). For this aim, we calculated spectra corresponding to the model of $T_{\mathrm{eff}}=7750$ $\mathrm{K}, \log (g)=4$, and $v \sin i=55 \mathrm{~km} / \mathrm{s}$ (Beskrovnaya et al., 1999). Errors in determining $E W\left(\mathrm{H}_{\alpha}\right)$ do not exceed $0.5 \AA$.

\section{Results of observations}

\subsection{The $\mathbf{H}_{\alpha}$ line}

Fig. 1 shows an example of the HD 36112 spectrum in the region of the $\mathrm{H}_{\alpha}$ emission line. This line is seen to represent broad emission, in whose blue wing there may be observed variable absorption components forming in the wind (P Cygtype profile). On some nights, two absorption components instead of one absorption could be observed. This allowed us to distinguish a low-velocity (line-of-sight velocities do not exceed $-200 \mathrm{~km} / \mathrm{s}$ ) and a high-velocity wind components. However, we cannot exclude the fact that the number of these components is greater.

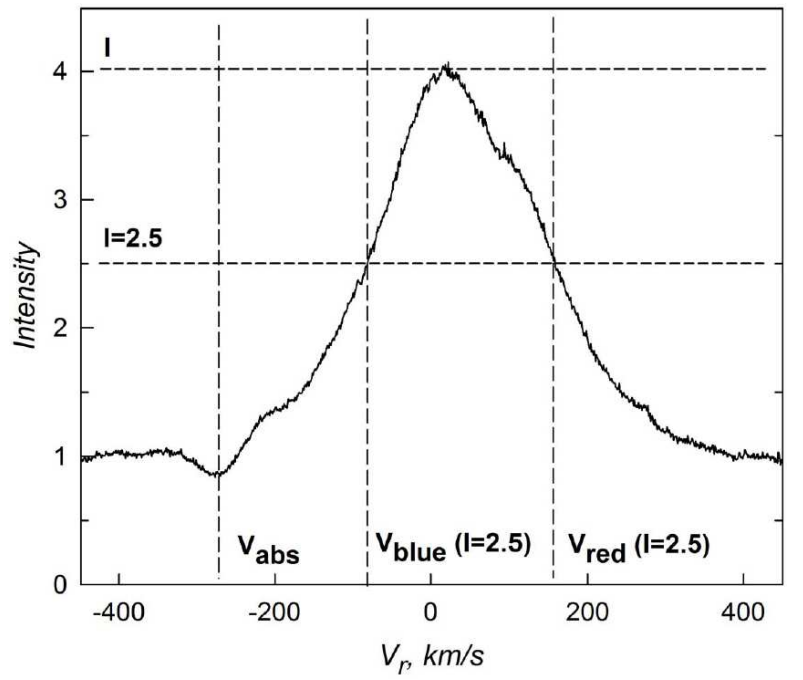

Fig. 1. An example of determining parameters of the $\mathrm{H}_{\alpha}$ emission line

To analyze many-year variability of emission, we singled out several parameters (see Fig. 1). These are the equivalent width of $E W\left(\mathrm{H}_{\alpha}\right)$ emission and its maximum intensity $I$, line-of-sight velocities of absorption components shifted to the blue side $V_{\mathrm{abs}}$ and their intensities $I_{\mathrm{abs}}$, and also line-ofsight velocities of the blue $V_{\text {blue }}$ and red $V_{\text {red }}$ boundaries of the emission profile for the levels of intensity $I=2.5$. Based on two latter parameters, we estimated the emission width $W_{\mathrm{em}}(I=2.5)$ and bisector values $V_{\text {bis }}(I=2.5)$. Variations of these values with time are shown in Fig. 2 and Fig. 3.

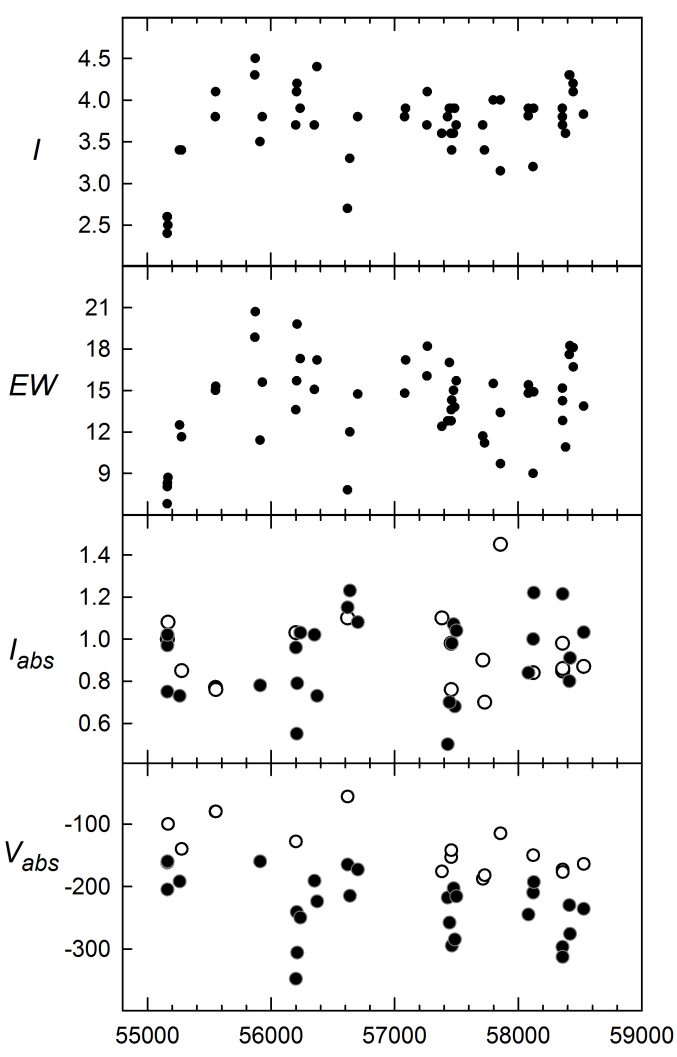

J.D. $2400000+$

Fig. 2. Many-year variability of parameters of the $\mathrm{H}_{\alpha}$ emission line: equivalent width $E W$, intensity $I$, and also line-of-sight velocities of wind components $V_{\text {abs }}$ and their intensities $I_{\text {abs }}$ (black circles denote high-speed wind components, empty circles denote low-speed ones)

In figures one can see noticeable variations in emission parameters from night to night. Such a variability type is described in detail in (Beskrovnaya et al., 1999) and associated with the inhomogeneous structure of the circumstellar gaseous envelope. Moreover, in variations of $I$ and $E W\left(\mathrm{H}_{\alpha}\right)$ one can notice a few minima of values observed at J.D. 2455100, 2456300, and 2458000 (see Fig. 2). Conversely, near J.D. 2456000, 2457200, and 2458300 there is a growth of these values. Interestingly, there simultaneously occurs an abrupt increase of velocities shifted to the blue side of absorption components $V_{\mathrm{abs}}$ and a growth of their depths (see variability $I_{\mathrm{abs}}$ ).

Analysis of Fig. 3 brings us to the same conclusion: on the dates of the highest values of $E W\left(\mathrm{H}_{\alpha}\right)$ and I there is an increase of line-of-sight velocities of the blue $V_{\text {blue }}(I=2.5)$ and red $V_{\text {red }}(I=2.5)$ boundaries of emission, i.e., on a time scale of 1200 days the emission width changes (see Fig. 3). Interestingly, we do not see any significant change on this 


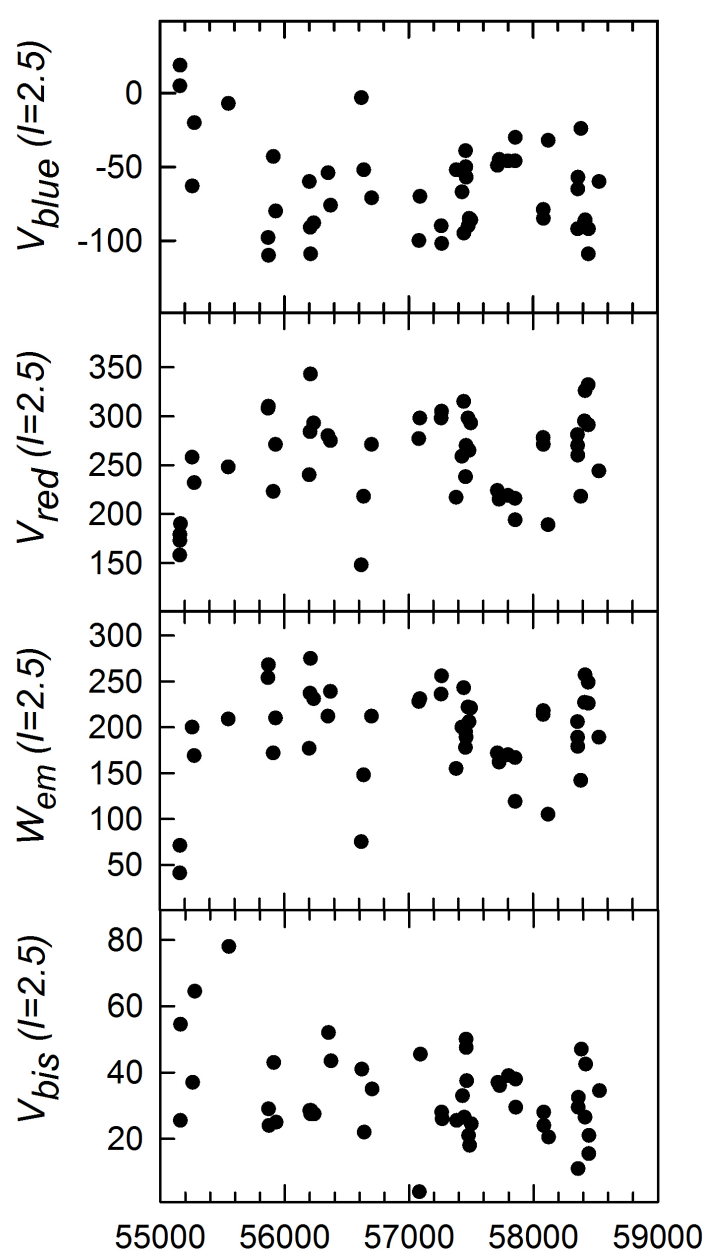

J.D. $2400000+$

Fig. 3. Many-year variability of parameters of the $\mathrm{H}_{\alpha}$ emission line: line-of-sight velocities of the blue $V_{\text {blue }}$ and red $V_{\text {red }}$ boundaries of the emission profile, the emission width $W_{\mathrm{em}}$, and the bisector position $V_{\text {bis }}$ for intensity levels $I=2.5$

scale in variations of the bisector value $V_{\text {bis }}(I=2.5)$. One can also assume the existence of one more variability scale. It looks like a many-year trend in variations of $E W\left(\mathrm{H}_{\alpha}\right)$ and $V_{\text {abs }}$ covering the whole period of observations (more than 4000 days).

Thus, the obtained results show the existence of some characteristic time (about $1200^{d}$ ), during which the physical and kinematic characteristics of outflowing CS matter vary in the circumstellar gaseous envelope of HD 36112. Apparently, we can talk about activity cycles in the course of which the process of gas outflow for the star (wind) either strengthens or weakens.

\subsection{NaI D resonance doublet lines}

Emission lines of the sodium resonance doublet observed for Ae/Be Herbig stars are weaker than the $\mathrm{H}_{\alpha}$ line. They have a quite symmetric profile, which, contrary to $\mathrm{H}_{\alpha}$ emission, is formed basically in the accretion disk rather than in the wind.
For the same reason, the NaI D emission lines are narrower, whereas the absorption components shifted to the blue side do not already fall into the region of the blue emission wing, as in hydrogen lines. This can be seen in Fig. 4, where as an example we show the spectrum of HD 36112 in the NaI D region derived on September 30, 2012.

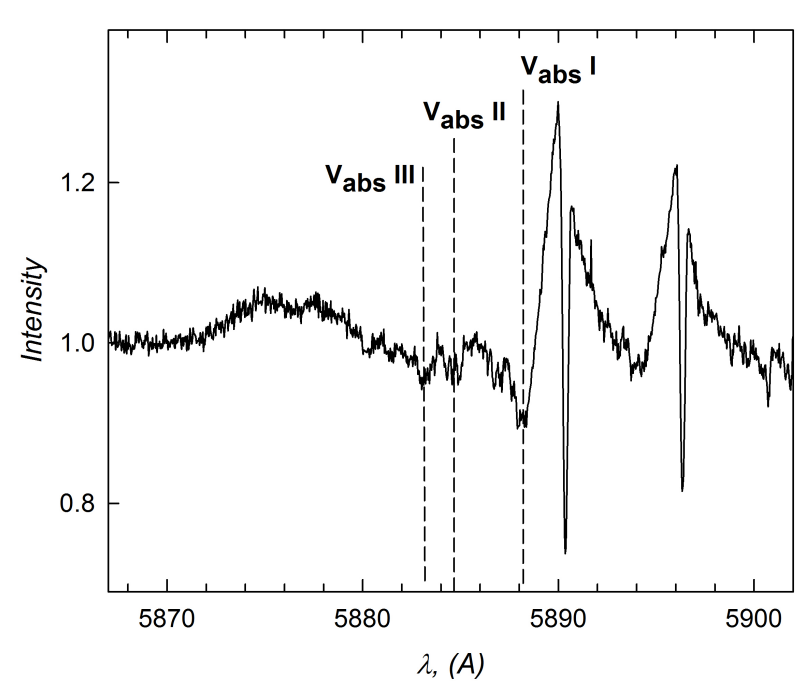

Fig. 4. An example of the HD 36112 spectrum in the sodium resonance doublet line region

Narrow interstellar absorption is seen well in broad doublet emission lines. On the blue side from emission one can see the CS absorption components forming, as in the $\mathrm{H}_{\alpha}$ line, in the wind. It is worth noting that here we already see three absorption components rather than two. The most lowvelocity of them has a velocity of $-90 \mathrm{~km} / \mathrm{s}$. In the $\mathrm{H}_{\alpha}$ line, lines with such velocities are basically not seen, since they fall into the region of strong intensity gradient.

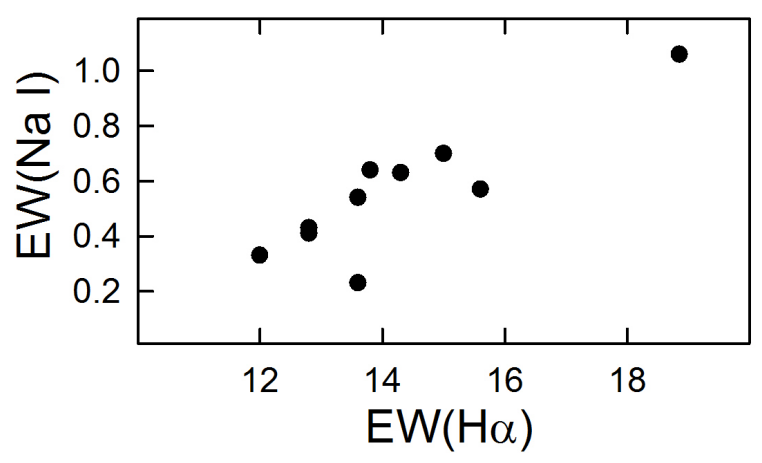

Fig. 5. Relation between the variation of equivalent widths $E W(\mathrm{NaI})$ and $E W\left(\mathrm{H}_{\alpha}\right)$

To analyze variability of the NaI D doublet lines, we determine the equivalent width of emission $E W(\mathrm{NaI} \mathrm{D})$ and line-of-sight velocities of wind components $V_{\text {abs }}(\mathrm{NaI})$. Unfortunately, there are too few spectra derived in this region, 


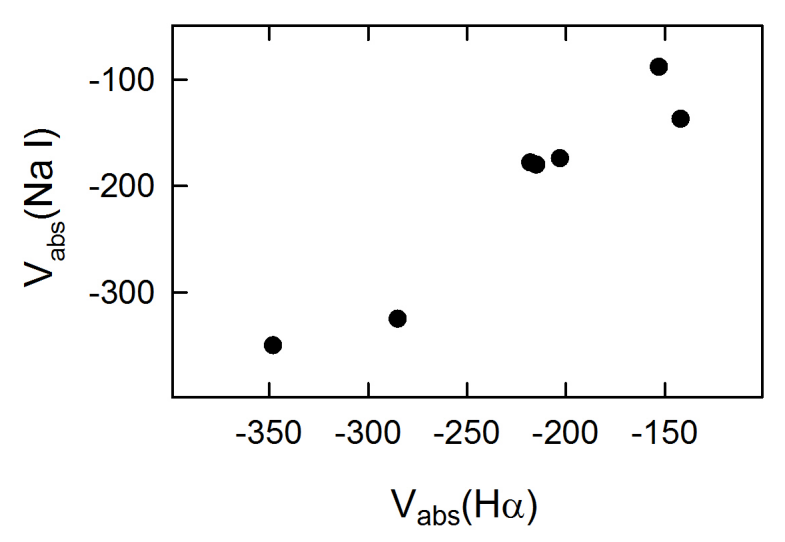

Fig. 6. Relation between the variation of line-of-sight velocities of wind components $V_{\mathrm{abs}}(\mathrm{NaI})$ and $V_{\mathrm{abs}}\left(\mathrm{H}_{\alpha}\right)$

therefore we will restrict ourselves to just a comparison of variability of equivalent widths and line-of-sight velocities with a variation of analogous parameters in the $\mathrm{H}_{\alpha}$ emission line. These results are shown in Fig. 5 and Fig. 6.

As seen from figures, there is a relation between the variability of $\mathrm{NaI} \mathrm{D}$ and $\mathrm{H}_{\alpha}$ lines, but the number of spectra in the sodium region does not yet allow us to confidently speak about a correlation between these lines. Nonetheless, such a correlation is fairly natural and expected (Kozlova et al., 2007a), since it is caused by a close relationship between the processes of accretion and outflow in young stars.

The confirmation of this correlation is of particular importance, since it will mean that the parameters of the NaI D doublet lines should also exhibit variability on a time scale of 1200 days. As stated above, the presence in the CS disk of a forming planet causes a variation of the accretion rate on the star on a time scale that is equal to the orbital period (Demidova, 2009). Since the NaI D emission lines are formed in the accretion disk, we can obtain a direct confirmation of the variation in the accretion rate for HD 36112.

\section{Conclusions}

As noted above, the data of many-year spectral monitoring of HD 36112 showed the presence of variability on several time scales. Of particular interest is a variability scale of about $1200^{d}$, on which very strong variations of parameters of the outflowing gas (wind) in the $\mathrm{H}_{\alpha}$ line occur. They show that the wind strengthens and weakens, and the picture turns out to be very similar to the spectral variability of the MWC 480 star (Kozlova et al., 2007a). The similarity is manifested in the presence in MWC 480 of the longer variability scale observed as a trend covering the whole period of observations (more than 4000 days). One more fact brings these stars together: no signatures of binarity were detected, but an active process of planet formation is observed.

We mentioned that the photometric variability of HD 36112 , due to the intermediate orientation of its disk with respect to an observer, does not exceed a few tenths of stellar magnitude. At the same time, there exists a group of young stars whose orientation is closer to the edge-on position.
These are UX Ori-type stars exhibiting very strong photopolarimetric activity due to eclipses produced by clouds of gas and dust (see, e.g., Rostopchina et al., 1999). Many-year photopolarimetric monitoring of these objects showed that brightness weakenings are not random but exhibit variability on a time scale of years. Whereas, for one star several such periods are revealed, the longest of them can reach several tens of years. Their availability shows the presence of stable large-scale inhomogeneities in the CS structure of gas and dust disks of these stars. Today, there are no doubts that such a structure is due to the planet formation process.

In this context, there must be pointed out the data of a spectral study of the CQ Tau star (Kozlova et al., 2007b). The shortest of photometric periods of the star accounts for about $1000^{d}$ (Shakhovskoi et al., 2005), which is close to the spectral variability scale of CQ Tau (about $700^{d}$ ). Moreover, the data of simultaneous spectral and photometric monitoring of this star showed that it was dust clouds causing brightness variations that, involving into the inner region of the accretion disk, produced the appearance of gas with anomalous kinematic characteristics and governed unusual spectral activity of the star. The relationship between photometric and spectral variability was considered in the context of the MWC 480 star (Kozlova et al., 2007a). Thus, we would like to hope that the HD 36112 star will also be included into the photometric monitoring.

All the stated above makes it possible to associate the results of many-year spectral monitoring of HD 36112 with the formation of massive planets in the inner region of its circumstellar disk. The spectral monitoring of this star is to be continued also in other spectral ranges.

The authors are grateful to the referee for interest to the article and useful remarks that to a great extent helped to improve the manuscript.

\section{References}

Alecian E., Wade G.A., Catala C., et al., 2013. Mon. Not. Roy. Astron. Soc., vol. 429, pp. 1001-1026.

Benisty M., Juhasz A., Boccaletti A., et al., 2015. Astron. Astrophys., vol. 578, p. L6.

Beskrovnaya N.G., Pogodin M.A., Miroshnichenko A.S., et al., 1999. Astron. Astrophys., vol. 343, pp. 163-174.

Boehler Y., Ricci L., Weaver E., et al., 2018. Astrophys. J., vol. 853, pp. 162-176.

Demidova T.V., 2016. Astrofizika, vol. 59, no. 4, pp. 505518. (In Russ.)

Demidova T.V., 2009. Astrofizika, vol. 52, no. 4, pp. 623630. (In Russ.)

Eisner J.A., Lane B.F., Hillenbrand L.A., Akeson R.L., Sargent A.I., 2004. Astrophysp. J., vol. 613, pp. 1049-1071.

Grinin V.P., Rostopchina A.N., 1996. Astronomy Rep., vol. 40 , iss. 2, p. 171-178.

Isella A., Tatulli E., Natta A., Testi L., 2008. Astron. Astrophys., vol. 483, p. L13.

Kozlova O.V., Alekseev I.Yu., Shakhovskoi D.N., 2007a. Astrofizika, vol. 50, no. 4, pp. 565-588. (In Russ.)

Kozlova O.V., Rostopchina A.N., Shakhovskoi D.N., Alekseev I.Yu., 2007b. Astrofizika, vol. 50, no. 1. pp. 39-56. (In Russ.) 
Palla F., Stahler W.S., 1993. Astrophys. J., vol. 418, pp. 414425.

Pogodin M.A., 1995. Astrophys. Space Sci., vol. 224, pp. 539-540.

Rostopchina A.N., et al., 1999. Pis'ma v Astron. zhurn., vol. 25, no. 4, pp. 291-298. (In Russ.)
Shakhovskoi D.N., Grinin V.P., Rostopchina A.N., 2005. Astrofizika, vol. 48, no. 2, pp. 165-174. (In Russ.)

The P.S., De Winter D., Perez M.R., 1994. Astron. Astropys. Suppl. Ser., vol. 104, pp. 315-339. 